\title{
Evolution of electron pitch angle distributions following injection from the plasma sheet
}

\author{
X. Tao, ${ }^{1}$ R. M. Thorne,${ }^{1}$ W. Li,${ }^{1}$ B. Ni, ${ }^{1}$ N. P. Meredith, ${ }^{2}$ and R. B. Horne ${ }^{2}$ \\ Received 26 October 2010; revised 27 January 2011; accepted 17 February 2011; published 28 April 2011.
}

[1] The temporal evolution of the phase space density of plasma sheet electrons $(100 \mathrm{eV}-$

$30 \mathrm{keV}$ ) injected into the nightside at $L=6$ during moderate geomagnetic activity is

investigated using a quasi-linear diffusion formulation. Scattering in energy and pitch angle during interactions with both whistler mode chorus waves and electron cyclotron harmonic waves are included using an improved wave model recently obtained using CRRES spacecraft data. We compare our simulation results with observations from the THEMIS spacecraft and demonstrate that the formation of the observed electron pitch angle distributions is mainly due to resonant interactions with a combination of upper and lower band chorus waves. The pancake distributions at low energies $(E<2 \mathrm{keV})$, the flattened pitch angle distributions at medium energies (between $2-3 \mathrm{keV}$ ), and the distributions with enhanced pitch angle anisotropy at high energies $(E>3 \mathrm{keV})$ are explained using the banded chorus wave structure with a power minimum at half the electron cyclotron frequency. Results of the current work can be used to model the dynamical evolution and resultant global distribution of plasma sheet electrons.

Citation: Tao, X., R. M. Thorne, W. Li, B. Ni, N. P. Meredith, and R. B. Horne (2011), Evolution of electron pitch angle distributions following injection from the plasma sheet, J. Geophys. Res., 116, A04229, doi:10.1029/2010JA016245.

\section{Introduction}

[2] Energetic $(\sim \mathrm{keV})$ plasma sheet electrons injected into the inner magnetosphere during active times act as an energy source for various processes in the inner magnetosphere. Both electromagnetic whistler mode chorus waves and electrostatic electron cyclotron harmonic (ECH) waves are enhanced on the nightside because of the free energy provided by the injected electrons [e.g., Horne et al., 1981, 2003; Li et al., 2008, 2009]. Whistler mode chorus waves are typically excited in two distinct bands with a power minimum around $0.5 \Omega_{e 0}$, where $\Omega_{e 0}$ is the equatorial electron cyclotron frequency [Tsurutani and Smith, 1974; Anderson and Maeda, 1977]. The origin of this power minimum is still under active research [e.g., Omura et al., 2009]. In contrast, ECH waves occur in bands between harmonics of $\Omega_{e 0}$, bounded below by $\Omega_{e 0}$ and above by the upper hybrid resonance frequency [Ashour-Abdalla and Kennel, 1978]. Both excited plasma waves interact with energetic electrons causing a portion of the plasma sheet electrons to be scattered into the atmosphere to form the diffuse aurora [Chen and Schulz, 2001; Horne et al., 2003; Ni et al., 2008; Meredith et al., 2009; Thorne et al., 2010; Ni et al., 2011a, 2011b]. Chorus is also responsible for local energization of electrons to $\mathrm{MeV}$ energy range in the outer

\footnotetext{
${ }^{1}$ Department of Atmospheric and Oceanic Sciences, University of California, Los Angeles, California, USA.

${ }^{2}$ British Antarctic Survey, Cambridge, UK.

Copyright 2011 by the American Geophysical Union. 0148-0227/11/2010JA016245
}

radiation belt [Horne and Thorne, 2003; Meredith et al., 2003; Albert, 2004; Shprits et al., 2006a; Li et al., 2007; Summers et al., 2007; Varotsou et al., 2008; Albert et al., 2009; Xiao et al., 2010], and for the generation of plasmaspheric hiss [Bortnik et al., 2008, 2009], which is responsible for the slot between the inner and outer radiation belt [Lyons and Thorne, 1973].

[3] Pancake distributions (peaked around $90^{\circ}$ ) of electrons, usually formed within a few hours after substorm injections [Meredith et al., 2000], have been observed in the energy range below a few $\mathrm{keV}$. Such distributions have long been considered to result from resonant interactions between energetic electrons and either ECH waves or chorus waves, because both waves are able to resonate with electrons between a few hundred $\mathrm{eV}$ to a few $\mathrm{keV}$, where pancake distributions are observed [Wrenn et al., 1979; Horne and Thorne, 2000; Meredith et al., 2000; Su et al., 2009]. However, a definite determination of which wave mode is more effective in producing pancake distributions has not previously been possible, due to a lack of information on the power spectral intensities of the two waves.

[4] In addition to the pancake distributions identified on the nightside using the Combined Release and Radiation Effects Satellite (CRRES) data [Meredith et al., 2000], additional features of the global distribution of plasma sheet electrons have been identified by Li et al. [2010a] using data from the Time History of Events and Macroscale Interactions during Substorms (THEMIS) spacecraft. Li et al. [2010a] demonstrated that lower-energy (from a few hundred $\mathrm{eV}$ to about $2 \mathrm{keV}$ ) electrons observed on the dayside persistently exhibit pronounced pancake distributions. Also 
the anisotropy of higher-energy $(>10 \mathrm{keV})$ electrons becomes greatly enhanced as injected electrons drift from the injection location on the nightside to the dayside, while their fluxes dramatically decrease, presumably due to pitch angle scattering by resonant waves. Between these two energy ranges, Li et al. [2010a] identified a population of electrons around a few $\mathrm{keV}$ that have a relatively flattened pitch angle distribution, in contrast to the highly anisotropic distributions at lower and higher energies. The cause of these different features of electron pitch angle distributions is the main topic of the current paper.

[5] Quasi-linear theory has been used by Su et al. [2009] to investigate the evolution of electron pitch angle distributions after substorm injections due to interactions with whistler mode chorus waves. They demonstrated that pancake distributions could form on a timescale of $5 \mathrm{~h}$ by interactions with chorus waves. However, three important features were not considered in their work. First, the high density approximation was used to calculate quasi-linear diffusion coefficients. However, as demonstrated by Glauert and Horne [2005, Figures 2 and 3], the high density approximation could cause serious errors at $10 \mathrm{keV}$, depending on the plasma density and the wave model used. Second, the model used for the chorus spectral properties was not directly based on observations. Third, only chorus waves were considered in their calculation, thus the importance of ECH waves in the formation of pancake distributions is still unknown.

[6] In section 2, we present observations from THEMIS showing typical electron pitch angle distributions observed at different magnetic local times (MLTs) after electron injections on the nightside. In section 3 , we model the evolution of electron pitch angle distributions using quasi-linear theory, with a greatly improved statistical nightside wave model recently obtained from CRRES data [Ni et al., 2011a, 2011b]. We explain different features of the evolving electron pitch angle distributions from simulation and compare them with observations from THEMIS in sections 3.1, 3.2, and 3.3. Finally, we discuss and summarize our results in section 4 .

\section{Observation From THEMIS}

[7] The THEMIS spacecraft [Angelopoulos, 2008] consists of five probes with near-equatorial orbits with apogees above $10 R_{E}$ and perigees below $2 R_{E}$. Each THEMIS probe contains an electrostatic analyzer (ESA), which provides electron pitch angle distributions in the energy range of a few eV up to $30 \mathrm{keV}$, and a Solid State Telescope (SST), which provides electron pitch angle distributions in the energy range of $25 \mathrm{keV}$ to $1 \mathrm{MeV}$. Electron phase space densities obtained from THEMIS data on the nightside will be used to provide initial conditions to the numerical simulation in section 3 .

[8] In Figure 1 we show electron pitch angle distributions at $L=6$ obtained from ESA that are typical both during (nightside) and after (later MLT) an electron injection event as electrons drift toward the dayside. The distribution during an injection event (Figure 1b) shows positive anisotropy at energies higher than about $0.7 \mathrm{keV}$, which could provide free energy for excitation of chorus waves [Horne et al., 2003; Li et al., 2008, 2009]. The distributions, shown in
Figure 1d, obtained at 04:00 MLT following an injection event (with $\mathrm{AE}$ index decreasing from about $500 \mathrm{nT}$ to about $100 \mathrm{nT}$ in the previous $3 \mathrm{~h}$ ) exhibit three distinct features. First, electrons with energies lower than about $1.28 \mathrm{keV}$ exhibit a typical pancake distribution that has been discussed in previous work [Meredith et al., 1999, 2000]. Second, for electrons from about $1.28 \mathrm{keV}$ to $2.22 \mathrm{keV}$, the pitch angle distributions are relatively flat. As discussed below, the exact energy range of electrons with this flat pitch angle distributions depends on parameters such as the ambient magnetic field strength and plasma density. Finally, higher-energy $(\gtrsim 3 \mathrm{keV})$ electrons exhibit enhanced anisotropy, as summarized by Li et al. [2010a]. Figure 1c, obtained at 06:36 MLT following an injection event (with $\mathrm{AE}$ index decreasing from about $1000 \mathrm{nT}$ to about $100 \mathrm{nT}$ in the previous $3 \mathrm{~h}$ ), shows roughly the same but more pronounced features than Figure 1d, indicating that the features described above are robust and probably continue to evolve as electrons are transported toward the dayside [ $\mathrm{Li}$ et al., 2010a]. For a more detailed statistical analysis of the observed electron pitch angle distributions following injections, we refer readers to $L i$ et al. [2010a]. In section 3 , we present a numerical simulation of the temporal evolution of the electron distribution due to interactions with waves observed on the nightside under moderate geomagnetic activity, and we compare this to the observed pitch angle distributions to explain the general features discussed above.

\section{Numerical Modeling Using Quasi-linear Theory}

[9] Quasi-linear theory is used to model the evolution of the pitch angle distribution of injected electrons due to interactions with chorus waves and ECH waves. We solve the following bounce-averaged equatorial pitch angle $\left(\alpha_{0}\right)$ and momentum $(p)$ diffusion equation for phase space density $(f)$ at $L=6$

$$
\begin{aligned}
\frac{\partial f}{\partial t}= & \frac{1}{G p} \frac{\partial}{\partial \alpha_{0}} G\left(D_{\alpha_{0} \alpha_{0}} \frac{1}{p} \frac{\partial f}{\partial \alpha_{0}}+D_{\alpha_{0} p} \frac{\partial f}{\partial p}\right) \\
& +\frac{1}{G} \frac{\partial}{\partial p} G\left(D_{\alpha_{0} p} \frac{1}{p} \frac{\partial f}{\partial \alpha_{0}}+D_{p p} \frac{\partial f}{\partial p}\right)-\frac{f}{\tau},
\end{aligned}
$$

where $D_{\alpha_{0} \alpha_{0}}, D_{\alpha 0 p}$ and $D_{p p}$ are bounce-averaged pitch angle, mixed and momentum diffusion coefficients. Here $G$ is a Jacobian factor, $G=p^{2} T\left(\alpha_{0}\right) \sin \left(\alpha_{0}\right) \cos \left(\alpha_{0}\right)$, and $T\left(\alpha_{0}\right) \approx$ $1.30-0.56 \sin \left(\alpha_{0}\right)$ is the normalized bounce period. The loss time $\tau$ is set to a quarter of the bounce period if $\alpha_{0}<\alpha_{\mathrm{L}}=2.85^{\circ}$ (the loss cone angle satisfying $\sin \alpha_{\mathrm{L}}=$ $\left.\mathrm{L}^{-3 / 2}(4-3 / \mathrm{L})^{-1 / 4}\right)$ and infinity otherwise.

[10] The diffusion coefficients in equation (1) have been evaluated using the spectral intensity of nightside whistler mode chorus emissions and ECH waves obtained from a recent statistical analysis of CRRES wave data under moderate geomagnetic conditions $\left(100<\mathrm{AE}^{*}<300 \mathrm{nT}\right)$, together with theoretical calculations of the wave normal distribution assuming a dipole magnetic field model at $L=6$ [Ni et al., 2011a, 2011b]. To calculate local quasi-linear diffusion coefficients of chorus waves, both the distribution of wave power $\left(B^{2}(\omega)\right)$ and wave normal angles $(g(X)$ with 

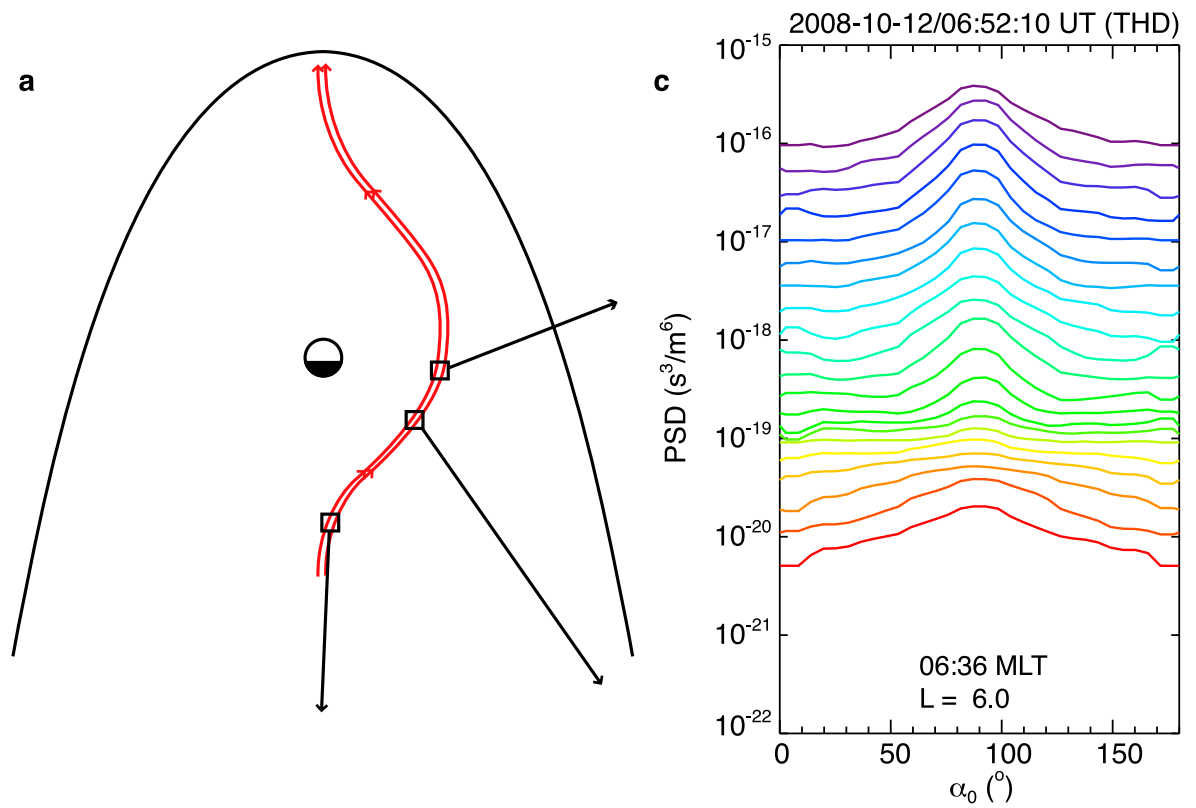

$0.11 \mathrm{keV}$

$0.14 \mathrm{keV}$

$0.19 \mathrm{keV}$

$0.25 \mathrm{keV}$

$0.32 \mathrm{keV}$

$0.43 \mathrm{keV}$

$0.56 \mathrm{keV}$

$0.74 \mathrm{keV}$

$0.98 \mathrm{keV}$

$1.28 \mathrm{keV}$

$1.69 \mathrm{keV}$
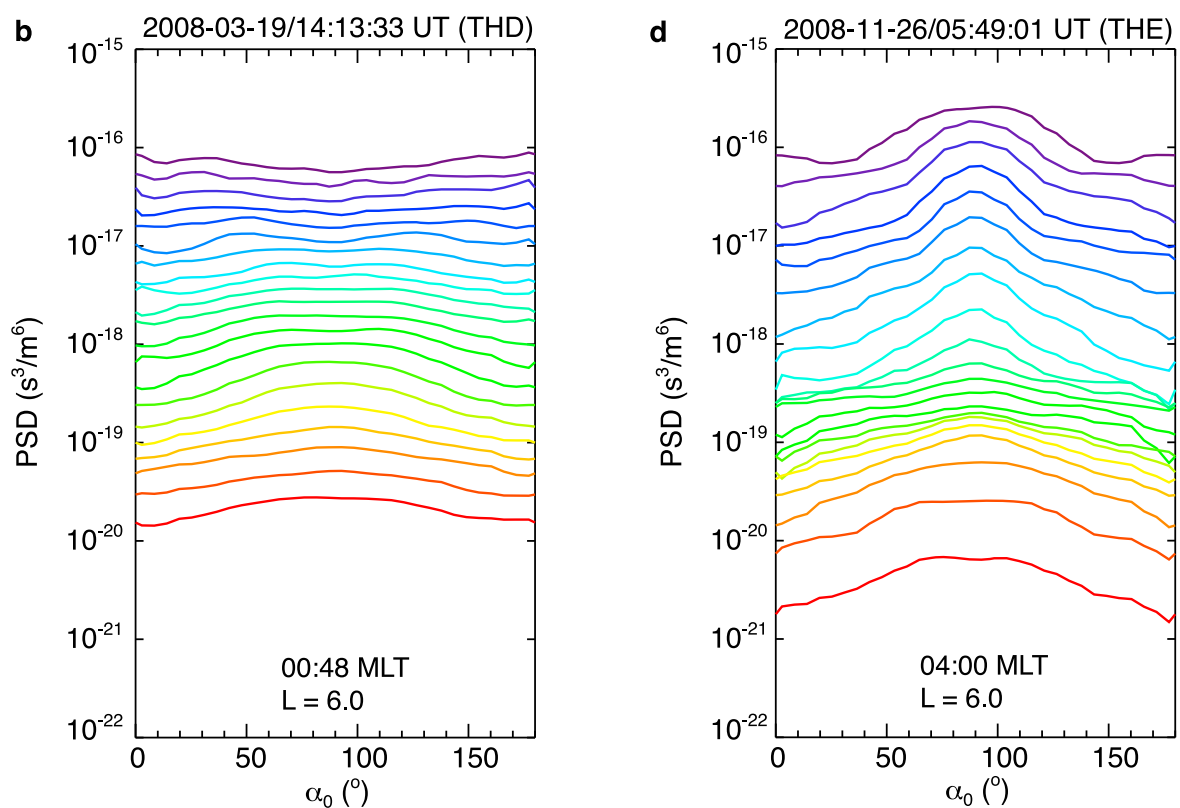

$2.22 \mathrm{keV}$

$2.93 \mathrm{keV}$

$3.85 \mathrm{keV}$

$5.07 \mathrm{keV}$

$6.68 \mathrm{keV}$

$8.79 \mathrm{keV}$

$11.6 \mathrm{keV}$

$15.2 \mathrm{keV}$

$20.0 \mathrm{keV}$

$26.4 \mathrm{keV}$

Figure 1. (a) A schematic plot showing drift paths (red lines) of plasma sheet electrons injected into the inner magnetosphere. Squares show the rough locations where measurements in Figures 1b, 1c, and 1d were taken. Representative electron phase space densities at different energies (represented by different colors) calculated using data from ESA of THEMIS D (THD) or E (THE) are shown for (b) 0048 MLT, (c) 0636 MLT, and (d) 0400 MLT.

$X \equiv \tan \theta$ and $\theta$ the wave normal angle) are assumed to be Gaussian [Lyons, 1974; Shprits and Ni, 2009], i.e.,

$$
B^{2}(\omega) \propto\left\{\begin{array}{cc}
\exp \left[-\frac{\left(\omega-\omega_{\mathrm{m}}\right)^{2}}{(\Delta \omega)^{2}}\right] & \omega_{\mathrm{LC}}<\omega<\omega_{\mathrm{UC}} \\
0 & \text { otherwise }
\end{array}\right.
$$

and

$$
g(X)=\left\{\begin{array}{cc}
\exp \left[-\frac{\left(X-X_{\mathrm{m}}\right)^{2}}{(\Delta X)^{2}}\right] & X_{\mathrm{LC}}<X<X_{\mathrm{UC}} \\
0 & \text { otherwise }
\end{array}\right.
$$

where subscript "UC" means upper cutoff and "LC" lower cutoff. The wave power peaks at $\omega_{\mathrm{m}}$ and $X_{\mathrm{m}}$, and has width $\Delta \omega$ and $\Delta X \equiv \tan (\Delta \theta)$. The parameters $\omega_{\mathrm{m}}$ and $\Delta \omega$ are obtained by performing a least squares Gaussian fit to CRRES data, while the wave normal angle distribution is chosen to be representative of previous theoretical and observational results [Ni et al., 2011b]. For ECH waves, the wave power $E^{2}(\omega)$ is also assumed to have a Gaussian as in equation (2), but the latitude-dependent wave normal angle distribution is obtained using ray-tracing results from the HOTRAY code [Horne, 1989]. The local diffusion coefficients are then bounce averaged using a dipole magnetic 
Table 1. Parameters of the Nightside Chorus Wave Model for Lower Band Chorus and Upper Band Chorus at $L=6$ Under Moderate Geomagnetic Activity

\begin{tabular}{|c|c|c|c|c|c|c|c|c|c|c|}
\hline & Latitude Range & $B_{w}(\mathrm{pT})$ & $\omega_{\mathrm{m}} / \Omega_{e}$ & $\Delta \omega / \Omega_{e}$ & $\omega_{\mathrm{LC}} / \Omega_{e}$ & $\omega_{\mathrm{UC}} / \Omega_{e}$ & $\theta_{\mathrm{m}}\left({ }^{\circ}\right)$ & $\Delta \theta\left(^{\circ}\right)$ & $\theta_{\mathrm{LC}}\left({ }^{\circ}\right)$ & $\theta_{\mathrm{UC}}\left({ }^{\circ}\right)$ \\
\hline & $|\lambda|<5^{\circ}$ & 20 & 0.28 & 0.12 & \multirow{3}{*}{0.1} & \multirow{3}{*}{0.5} & 0 & 30 & 0 & 50 \\
\hline \multirow[t]{2}{*}{ LBC } & $5^{\circ}<|\lambda|<10^{\circ}$ & 35 & 0.3 & 0.08 & & & 15 & 30 & 0 & 50 \\
\hline & $10^{\circ}<|\lambda|<15^{\circ}$ & 20 & 0.26 & 0.07 & & & 30 & 30 & 0 & 50 \\
\hline \multirow[t]{2}{*}{ UBC } & $|\lambda|<5^{\circ}$ & 15 & 0.54 & 0.1 & \multirow[t]{2}{*}{0.5} & \multirow[t]{2}{*}{0.68} & 0 & 30 & 0 & 44 \\
\hline & $5^{\circ}<|\lambda|<10^{\circ}$ & 10 & 0.57 & 0.04 & & & 30 & 30 & 0 & 44 \\
\hline
\end{tabular}

field. We refer readers to Ni et al. [2011a, 2011b] for details regarding the wave modeling. Relevant parameters for whistler mode chorus waves are given in Table 1 and ECH waves in Table 2. The Full Diffusion Code [Shprits and Ni, 2009] is used to calculate the quasi-linear diffusion coefficients, shown in Figure 2, using the above wave model. The most striking feature of Figure 2 is that ECH waves can only resonate with electrons over a limited energy range and for pitch angles below about $20^{\circ}$. In contrast, combination of upper and lower band chorus waves can resonate with electrons over a broad range of pitch angles at energies from $30 \mathrm{eV}$ to a few hundred $\mathrm{keV}$. The total diffusion coefficients (lower panels) show a pronounced gap around a few $\mathrm{keV}$, which results from the chorus wave power gap around $\Omega_{e 0} / 2$.

[11] Given bounce-averaged diffusion coefficients, various numerical techniques have been developed or introduced to solve the diffusion equation (1) to provide accurate solutions [e.g., Albert and Young, 2005; Tao et al., 2008, 2009; Xiao et al., 2009]. We have developed a code at UCLA to numerically solve equation (1) using the Alternative Direction Implicit method described by Xiao et al. [2009] because the method is efficient and easy to code. We model the evolution of the injected plasma sheet electron distribution over an hour, which is the typical time needed for electrons to drift from the injection location on the nightside to dawn for a realistic enhanced convection electric field during moderately disturbed times [Chen and Schulz, 2001]. The initial condition is taken from THEMIS observation near local midnight, shown in Figures $1 \mathrm{~b}$ and $3 \mathrm{a}$. Note that probably due to the limited pitch angle resolution of the particle data from THEMIS, the electron phase space density inside the loss cone is not properly resolved, but this should not affect our main results below. We set $f$ to be constant at $30 \mathrm{eV}$ and $1 \mathrm{MeV}$, because the evolutionary timescale of these electrons is much longer
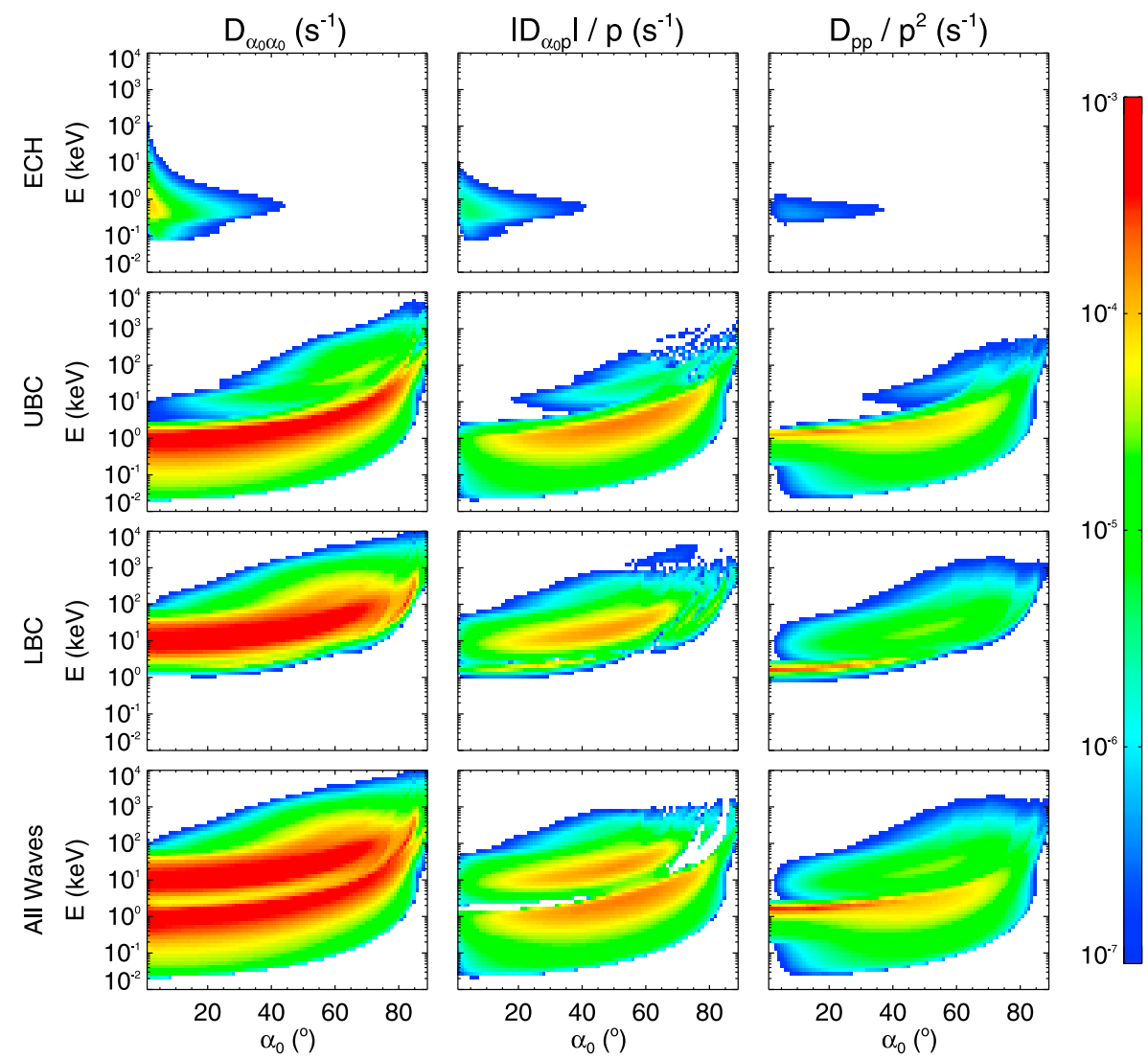

Figure 2. Pitch angle (left column), mixed (middle column), and momentum (right column) diffusion coefficients for ECH (first row), upper band chorus (the second row), lower band chorus (the third row), and all waves combined (fourth row). 
Table 2. Parameters of the Nightside ECH Wave Model at $L=6$ Under Moderate Geomagnetic Activity

\begin{tabular}{cccccc}
\hline ECH Band & $E_{w}(\mathrm{mV} / \mathrm{m})$ & $\omega_{\mathrm{m}} / \Omega_{e}$ & $\Delta \omega / \Omega_{e}$ & $\omega_{\mathrm{LC}} / \Omega_{e}$ & $\omega_{\mathrm{UC}} / \Omega_{e}$ \\
\hline 1 & 1.3 & 1.6 & 0.15 & 1.1 & 1.9 \\
2 & 0.5 & 2.55 & 0.15 & 2.1 & 2.9 \\
3 & 0.7 & 3.4 & 0.25 & 3.1 & 3.9 \\
4 & 0.8 & 4.2 & 0.25 & 4.1 & 4.9 \\
\hline
\end{tabular}

than a few hours, as can be inferred from the diffusion coefficients. We choose $\partial f / \partial \alpha_{0}=0$ at $\alpha_{0}=0^{\circ}$ and $90^{\circ}$, because pitch angle diffusion rates may at times exceed the strong diffusion limit [Schulz and Lanzerotti, 1974, p. 77].

[12] The evolution of the electron pitch angle distribution from our numerical simulation is shown is Figure 3. The top row exhibits the evolution of the electron distribution due to resonant interaction with all three waves, while the bottom row shows the evolved distribution after $1 \mathrm{~h}$ of scattering by $\mathrm{ECH}$ waves alone, upper band chorus (UBC) alone, and lower band chorus (LBC) alone. Clearly ECH waves only affect the phase space density of electrons over a limited energy range with pitch angles $\left(<20^{\circ}\right)$ near the loss cone, while chorus waves can modify the pitch angle distribution of electrons over a much broader energy and pitch angle range. Consequently, chorus waves are the main contributor for scattering electrons into the atmosphere to form the diffuse aurora [Thorne et al., 2010] around $L=6$. Here we demonstrate that they are also the main cause of the observed electron pitch angle distribution left behind in space following loss into the atmosphere. Our modeling does not include the effects of scattering near $90^{\circ}$ pitch angle due to processes such as bounce resonance [Shprits, 2009; Schulz and Lanzerotti, 1974, p. 62] or transit time scattering by magnetosonic waves [Bortnik and Thorne, 2010]. As a consequence, the simulations exhibit anomalous small bumps around $90^{\circ}$, which would probably be removed by this additional scattering. A test run (not shown) by setting $D_{\alpha 0 p}=0$ exhibits no anomalous enhancements, indicating that these anomalous enhancements result from coupling between pitch angle scattering and energy diffusion. But aside from this artifact, the topology of the electron pitch angle distribution obtained from the simulation after $1 \mathrm{~h}$ of wave scattering is very similar to that from THEMIS observation as discussed below.

\subsection{Pancake Distributions Below a Few keV}

[13] At energies below a few $\mathrm{keV}$, the simulated electron evolution exhibits pancake distributions [Wrenn et al., 1979], strongly peaked around $90^{\circ}$, similar to observations shown in Figure 1 and the earlier CRRES observations reported by Meredith et al. [1999]. The formation of the pancake distributions at lower energies is mainly a result of interactions with upper band chorus. To further illustrate the scattering process, we show diffusion coefficients in blue and the evolution of the electron phase space density at an energy of $0.98 \mathrm{keV}$ in black in Figure 4 (left). To provide a simple explanation for the origin of the pancake distributions, we ignore the contribution of mixed terms $\left(D_{\alpha_{0} p}\right)$. Note that the pitch angle diffusion coefficient is about 2 orders of magnitude higher at lower pitch angles than at pitch angles near $75^{\circ}$, which causes the rapid loss of low energy electrons over a broad pitch angle range between the loss cone and $\sim 60^{\circ}$ on a timescale comparable to an hour. Changes in the phase space density at higher pitch angles are much slower, leading to the evolution of pancake distributions. Thus as electrons drift toward the dayside after an

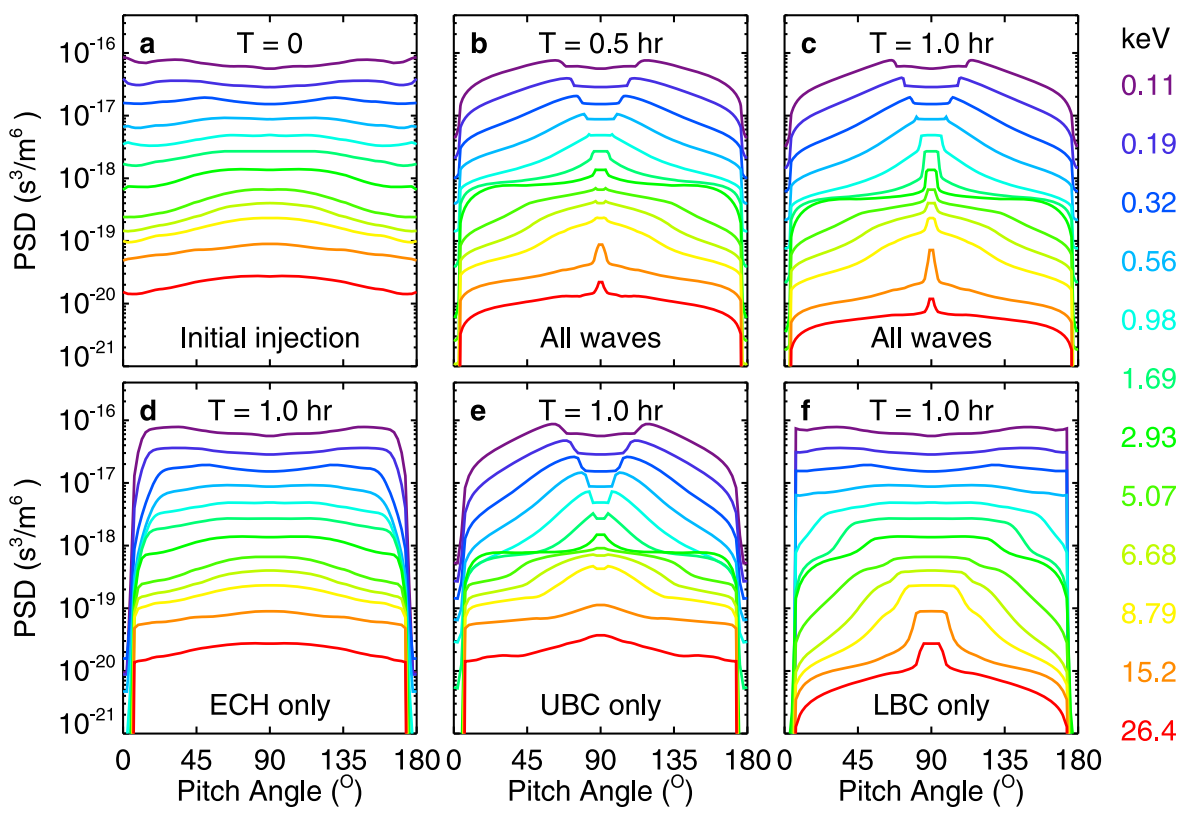

Figure 3. (a) Initial distribution of electrons following an injection and the evolution of electron distribution after interactions with the combination of ECH waves, upper band chorus, and lower band chorus for (b) $0.5 \mathrm{~h}$ and (c) $1 \mathrm{~h}$. To demonstrate the effect of each kind of waves on the electron distribution, we show the distribution of electrons after interactions for $1.0 \mathrm{~h}$ with (d) ECH waves only, (e) upper band chorus only, and (f) lower band chorus only. 

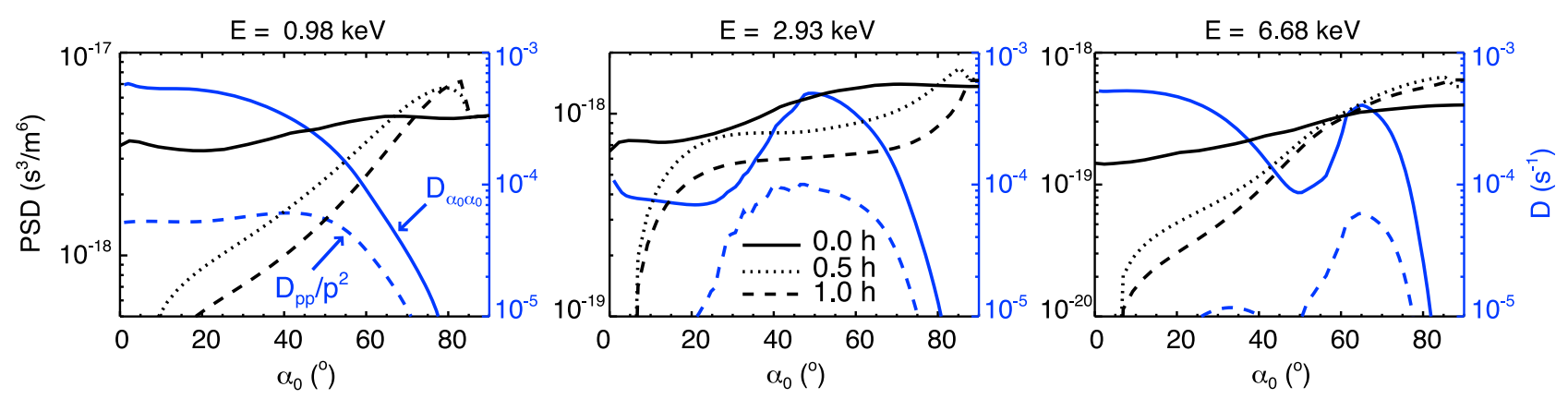

Figure 4. Diffusion coefficients $D_{\alpha_{0} \alpha_{0}}$ and $D_{p p} / p^{2}$ at a given energy and the corresponding evolution of the electron phase space density at (left) $E=0.98 \mathrm{keV}$, (middle) $2.93 \mathrm{keV}$, and (right) $6.68 \mathrm{keV}$. Phase space densities are shown at $t=0.0$ (solid lines), $0.5 \mathrm{~h}$ (dotted lines), and $1.0 \mathrm{~h}$ (dashed lines).

injection at night, the loss of electrons at lower pitch angles by scattering primarily from upper band chorus waves forms the pancake distribution on a timescale of about $1 \mathrm{~h}$.

\subsection{Flattened Distributions Near a Few keV}

[14] Around 2 to $3 \mathrm{keV}$, there is a pronounced flattening in both the pitch angle distribution and the energy distribution. The energy of electrons with a flattened distribution corresponds to the lowest cyclotron and Landau resonant energies with chorus waves near $0.5 \Omega_{e 0}$. While the Landau resonance contributes to the formation of a plateau in the energy distribution [Nicholson, 1992] shown in Figure 3, the pronounced isotropization in the pitch angle distribution is exhibited in Figure 4(middle), which shows $D_{\alpha_{0} \alpha_{0}}, D_{p p} / p^{2}$, and the evolution of the electron phase space density at $2.93 \mathrm{keV}$. At this energy, there is a pronounced reduction in $D_{\alpha_{0} \alpha_{0}}$ near the loss cone, significantly reducing the loss rate of electrons [Shprits et al., 2006b], while the peak of $D_{\alpha_{0} \alpha_{0}}$ indicates a rapid isotropization of the electron pitch angle distributions between $30^{\circ}$ to $70^{\circ}$. As noted earlier, the reduction in $D_{\alpha_{0} \alpha_{0}}$ near the loss cone results from the chorus wave power gap around $0.5 \Omega_{e 0}$, while the strong scattering at medium pitch angles comes from resonant interactions with upper band chorus. Thus the flattened distributions near a few $\mathrm{keV}$, which are a persistent feature of observed electron distributions at later MLT (Figures 1c and 1d), mainly result from the combined effect of reduced rates of loss to the atmosphere due to the wave power gap in chorus near $0.5 \Omega_{e 0}$ and rapid scattering by upper band chorus at intermediate pitch angles.

[15] Another consequence of the reduced loss rates for electrons at these energies is the reduced flux of electrons precipitating into the atmosphere. This can also be inferred from Figure 3, which shows that electron phase space density for $2 \sim 3 \mathrm{keV}$ electrons only decreases by about a factor of two, while the phase space densities at lower $(0.1 \sim$ $1 \mathrm{keV})$ and higher energies $(3 \sim 15 \mathrm{keV})$ decrease by about an order of magnitude at low pitch angles. This result is consistent with the observation shown by Miyoshi et al. [2010, Figure 3], which demonstrated a gap in the precipitating electron energy flux around a few keV. Our simulation suggests that this gap is caused by the reduced pitch angle scattering rates near the loss cone, which results from the chorus wave power gap around $0.5 \Omega_{e 0}$.

\subsection{Enhanced Anisotropy Above a Few keV}

[16] Above a few $\mathrm{keV}$, our simulations show that the anisotropy of electrons is increased after $1 \mathrm{~h}$ of scattering due to a decrease of phase space density at lower pitch angles and an increase of phase space density at higher pitch angles. Figure 4 (right) shows $D_{\alpha_{0} \alpha_{0}}, D_{p p} / p^{2}$, and the evolution of the electron phase space density at $6.68 \mathrm{keV}$. There is a deep minimum in $D_{\alpha_{0} \alpha_{0}}$ around $50^{\circ}$, which is caused by the wave power gap around $0.5 \Omega_{e 0}$. The main contribution to $D_{\alpha_{0} \alpha_{0}}$ is due to cyclotron resonant scattering by lower band chorus at pitch angles below $50^{\circ}$, while the peaks in $D_{\alpha_{0} \alpha_{0}}$ and $D_{p p}$ around $65^{\circ}$ are due to upper band chorus. The deep minimum of $D_{\alpha_{0} \alpha_{0}}$, called a "bottleneck" by Schulz [1991], slows the transport of electrons from high pitch angles to low pitch angles [Albert and Shprits, 2009]. As a consequence, energy diffusion from upper band chorus causes the increase in electron phase space density at $\alpha_{0}>50^{\circ}$, while lower band chorus causes rapid loss of electrons at $\alpha_{0}<40^{\circ}$ over a time scale comparable to an hour. The net effect leads to an enhancement of the electron pitch angle anisotropy consistent with the observations shown in Figure 1.

\section{Discussion and Summary}

[17] We have used the 2D bounce-averaged quasi-linear diffusion equation, with diffusion coefficients calculated using a new wave model from CRRES observations under moderately disturbed geomagnetic conditions, to model the evolution of electron phase space density following injections from the plasma sheet at night. It is generally thought that chorus waves are generated via a combination of linear amplification [Li et al., 2008, 2009] and a nonlinear process [Trakhtengerts, 1995; Nunn et al., 1997; Omura et al., 2008] with free energy from the injected electron distribution near a few $\mathrm{keV}$. This nonlinear process, however, cannot be treated using the quasi-linear theory and is not included in our modeling above.

[18] Despite our neglect of nonlinear effects, our simulation results reproduce the dominant features of the injected electrons observed on THEMIS and shown in Figure 1. We demonstrate that ECH waves can only resonate with electrons over a limited range of energies and pitch angles $\left(<20^{\circ}\right)$. Consequently ECH waves are not responsible for producing pancake distributions near $L=6$. Instead, our results show that a combination of upper and lower band 
chorus waves are the main driver of the formation of the observed pancake distributions (for $E \lesssim 2 \mathrm{keV}$ ), the flattened distributions (for $2 \lesssim E \lesssim 3 \mathrm{keV}$ ), and the distributions with enhanced anisotropy (for $E \gtrsim 3 \mathrm{keV}$ ). The resulting electron pitch angle distributions are closely related to the observed chorus wave power structure: a wave power gap around $0.5 \Omega_{e 0}$ divides chorus waves into an upper band and a lower band. We demonstrate that, because of the rapid loss at lower pitch angles from scattering by upper band chorus waves, lower energy $(E \lesssim 2 \mathrm{keV})$ electrons tend to form pancake distributions. The dual-band structure of chorus results in a significant decrease in $D_{\alpha_{0} \alpha_{0}}$ for certain energies at different pitch angles. At about $2 \sim$ $3 \mathrm{keV}$, the decrease of $D_{\alpha_{0} \alpha_{0}}$ occurs near the loss cone angle, thus dramatically reducing the loss rate of these electrons, forming a drop in precipitating electron fluxes reported by Miyoshi et al. [2010]. The pitch angle scattering at higher pitch angles from upper band chorus quickly flattens the pitch angle distribution, resulting in flattened pitch angle distributions for electrons at $2 \sim 3 \mathrm{keV}$. The location of the decrease of $D_{\alpha 0 \alpha 0}$ moves to higher pitch angles at higher energies, forming a "bottleneck" in pitch angle scattering which acts to enhance the pitch angle anisotropy of electrons with $E \geq 3 \mathrm{keV}$, due to the rapid loss from scattering by lower band chorus at lower pitch angles and energization by upper band chorus at higher pitch angles. Overall, our results explain the main observed features of the electron distribution following an injection. While the formation of the chorus wave power minimum at $0.5 \Omega_{e 0}$ is still under intensive research, our results demonstrated its important role in the evolution of electron pitch angle distributions after injections. Also because of the strong dependence of the resulting pitch angle distributions on chorus power spectrum structure, our results should be robust when the dominant scattering is by chorus waves. A different activity level or L shell might change the exact resonant energy of electrons, but not the three general features discussed above.

[19] Significant energization of the injected $(>3 \mathrm{keV})$ electron population occurs on timescales shorter than the transport time to the dayside ( $\sim$ a few hours). Consequently, injected plasma sheet electrons on open drift trajectories outside the Alfvén layer could be energized sufficiently to place them closer to or even within the Alfvén layer at the enhanced energy level. This energization process, together with fluctuations in the convection electric field [ $\mathrm{Li}$ et al., 2010b], can place the injected electrons on closed drift trajectories, thus forming an important source population for the trapped radiation belts. Such effects need to be incorporated into models and radiation belt dynamics.

[20] Acknowledgments. This work was supported in part by NSF grant ATM 0802843 and Heliophysics Theory grant NNX08A135G. We acknowledge NASA contract NAS5-02099 and V. Angelopoulos for use of data from the THEMIS Mission, specifically, C. W. Carlson and J. P. McFadden for use of ESA data and D. Larson and R. P. Lin for use of SST data.

[21] Philippa Browning thanks Michael Denton and another reviewer for their assistance in evaluating this manuscript.

\section{References}

Albert, J. M. (2004), Using quasi-linear diffusion to model acceleration and loss from wave-particle interactions, Space Weather, 2, S09S03, doi:10.1029/2004SW000069.
Albert, J. M., and Y. Y. Shprits (2009), Estimates of lifetimes against pitch angle diffusion, J. Atmos. Sol. Terr. Phys., 71, 1647-1652, doi:10.1016/j. jastp.2008.07.004.

Albert, J. M., and S. L. Young (2005), Multidimensional quasi-linear diffusion of radiation belt electrons, Geophys. Res. Lett., 32, L14110, doi:10.1029/2005GL023191.

Albert, J. M., N. P. Meredith, and R. B. Horne (2009), Three-dimensional diffusion simulation of outer radiation belt electrons during the 9 October 1990 magnetic storm, J. Geophys. Res., 114, A09214, doi:10.1029/ 2009JA014336.

Anderson, R. R., and K. Maeda (1977), VLF emissions associated with enhanced magnetospheric electrons, J. Geophys. Res., 82(1), 135-146, doi:10.1029/JA082i001p00135.

Angelopoulos, V. (2008), The THEMIS mission, Space Sci. Rev., 141, 5-34, doi:10.1007/s11214-008-9336-1.

Ashour-Abdalla, M., and C. F. Kennel (1978), Nonconvective and convective electron cyclotron harmonic instabilities, J. Geophys. Res., 83(A4), 1531-1543.

Bortnik, J., and R. M. Thorne (2010), Transit time scattering of energetic electrons due to equatorially confined magnetosonic waves, J. Geophys. Res., 115, A07213, doi:10.1029/2010JA015283.

Bortnik, J., R. M. Thorne, and N. P. Meredith (2008), The unexpected origin of plasmaspheric hiss from discrete chorus emissions, Nature, 452, 62-66, doi:10.1038/nature06741.

Bortnik, J., W. Li, R. M. Thorne, V. Angelopoulos, C. Cully, J. Bonnell, O. L. Contel, and A. Roux (2009), An observation linking the origin of plasmaspheric hiss to discrete chorus emissions, Science, 324(5928), 775-778, doi:10.1126/science. 1171273 .

Chen, M. W., and M. Schulz (2001), Simulations of storm time diffuse aurora with plasma sheet electrons in strong pitch angle diffusion, J. Geophys. Res., 106(A2), 1873-1886.

Glauert, S. A., and R. B. Horne (2005), Calculation of pitch angle and energy diffusion coefficients with the PADIE code, J. Geophys. Res., 110, A04206, doi:10.1029/2004JA010851.

Horne, R. B. (1989), Path-integrated growth of electrostatic waves: The generation of terrestrial myriametric radiation, J. Geophys. Res., 94(A7), 8895-8909.

Horne, R. B., and R. M. Thorne (2000), Electron pitch angle diffusion by electrostatic electron cyclotron harmonic waves: The origin of pancake distributions, J. Geophys. Res., 105(A3), 5391-5402.

Horne, R. B., and R. M. Thorne (2003), Relativistic electron acceleration and precipitation during resonant interactions with whistler-mode chorus, Geophys. Res. Lett., 30(10), 1527, doi:10.1029/2003GL016973.

Horne, R. B., P. J. Christiansen, M. P. Gough, K. Rönnmark, J. F. E. Johnson, J. Sojka, and G. L. Wrenn (1981), Amplitude variations of electron cyclotron harmonic waves, Nature, 294, 338-340.

Horne, R. B., R. M. Thorne, N. P. Meredith, and R. R. Anderson (2003), Diffuse auroral electron scattering by electron cyclotron harmonic and whistler mode waves during an isolated substorm, J. Geophys. Res., 108(A7), 1290, doi:10.1029/2002JA009736.

Li, W., Y. Y. Shprits, and R. M. Thorne (2007), Dynamic evolution of energetic outer zone electrons due to wave-particle interactions during storms, J. Geophys. Res., 112, A10220, doi:10.1029/2007JA012368.

Li, W., R. M. Thorne, N. P. Meredith, R. B. Horne, J. Bortnik, Y. Y. Shprits, and B. Ni (2008), Evaluation of whistler mode chorus amplification during an injection event observed on CRRES, J. Geophys. Res., 113, A09210, doi:10.1029/2008JA013129.

Li, W., R. M. Thorne, V. Angelopoulos, J. W. Bonnell, J. P. McFadden, C. W. Carlson, O. LeContel, A. Roux, K. H. Glassmeier, and H. U. Auster (2009), Evaluation of whistler-mode chorus intensification on the nightside during an injection event observed on the THEMIS spacecraft, J. Geophys. Res., 114, A00C14, doi:10.1029/ 2008JA013554.

Li, W., et al. (2010a), THEMIS analysis of observed equatorial electron distributions responsible for the chorus excitation, J. Geophys. Res. 115, A00F11, doi:10.1029/2009JA014845.

Li, W., R. M. Thorne, J. Bortnik, Y. Nishimura, V. Angelopoulos, L. Chen, J. P. McFadden, and J. W. Bonnell (2010b), Global distributions of suprathermal electrons observed on THEMIS and potential mechanisms for access into the plasmasphere, J. Geophys. Res., 115, A00J10, doi:10.1029/2010JA015687.

Lyons, L. R. (1974), Pitch angle and energy diffusion coefficients from resonant interactions with ion-cyclotron and whistler waves, J. Plasma Phys., 12, 417-432.

Lyons, L. R., and R. M. Thorne (1973), Equilibrium structure of radiation belt electrons, J. Geophys. Res., 78(13), 2142-2149.

Meredith, N. P., A. D. Johnstone, S. Szita, R. B. Horne, and R. R. Anderson (1999), "Pancake" electron distributions in the outer radia- 
tion belts, J. Geophys. Res., 104(A6), 12,431-12,444, doi:10.1029/ 1998JA900083.

Meredith, N. P., R. B. Horne, A. D. Johnstone, and R. R. Anderson (2000), The temporal evolution of electron distributions and associated wave activity following substorm injections in the inner magnetosphere, J. Geophys. Res., 105(A6), 12,907-12,917.

Meredith, N. P., M. Cain, R. B. Horne, R. M. Thorne, D. Summers, and R. R. Anderson (2003), Evidence for chorus-driven electron acceleration to relativistic energies from a survey of geomagnetically disturbed periods, J. Geophys. Res., 108(A6), 1248, doi:10.1029/2002JA009764.

Meredith, N. P., R. B. Horne, R. M. Thorne, and R. R. Anderson (2009), Survey of upper band chorus and ECH waves: Implications for the diffuse aurora, J. Geophys. Res., 114, A07218, doi:10.1029/2009JA014230.

Miyoshi, Y., Y. Katoh, T. Nishiyama, T. Sakanoi, K. Asamura, and M. Hirahara (2010), Time of flight analysis of pulsating aurora electrons, considering wave-particle interactions with propagating whistler mode waves, J. Geophys. Res., 115, A10312, doi:10.1029/2009JA015127.

Ni, B., R. M. Thorne, Y. Y. Shprits, and J. Bortnik (2008), Resonant scattering of plasma sheet electrons by whistler-mode chorus: Contribution to diffuse auroral precipitation, Geophys. Res. Lett., 35, L11106, doi:10.1029/2008GL034032.

Ni, B., R. M. Thorne, R. B. Horne, N. P. Meredith, Y. Y. Shprits, L. Chen, and W. Li (2011a), Resonant scattering of plasma sheet electrons leading to diffuse auroral precipitation: 1. Evaluation for electrostatic electron cyclotron harmonic waves, J. Geophys. Res., doi:10.1029/ 2010JA016232, in press.

Ni, B., R. M. Thorne, N. P. Meredith, R. B. Horne, and Y. Y. Shprits (2011b), Resonant scattering of plasma sheet electrons leading to diffuse auroral precipitation: 2. Evaluation for whistler-mode chorus waves, J. Geophys. Res., doi:10.1029/2010JA016233, in press.

Nicholson, D. R. (1992), Introduction to Plasma Theory, Krieger, Malabar, Fla.

Nunn, D., Y. Omura, H. Matsumoto, I. Nagano, and S. Yagitani (1997), The numerical simulation of VLF chorus and discrete emissions observed on the Geotail satellite using a Vlasov code, J. Geophys. Res., 102(A12), 27,083-27,097.

Omura, Y., Y. Katoh, and D. Summers (2008), Theory and simulation of the generation of whistler-mode chorus, J. Geophys. Res., 113, A04223, doi:10.1029/2007JA012622.

Omura, Y., M. Hikishima, Y. Katoh, D. Summers, and S. Yagitani (2009), Nonlinear mechanisms of lower-band and upper-band VLF chorus emissions in the magnetosphere, J. Geophys. Res., 114, A07217, doi:10.1029/ 2009JA014206.

Schulz, M. (1991), The magnetosphere, in Geomagnetism, vol. 4, edited by J. A. Jacobs, pp. 87-293, Academic, San Diego, Calif.

Schulz, M., and L. J. Lanzerotti (1974), Particle diffusion in the radiation belts, in Physics and Chemistry in Space, vol. 7, p. 77, Springer, Berlin.

Shprits, Y. Y. (2009), Potential waves for pitch angle scattering of nearequatorially mirroring energetic electrons due to the violation of the second adiabatic invariant, Geophys. Res. Lett., 36, L12106, doi:10.1029/2009GL038322.
Shprits, Y. Y., and B. Ni (2009), Dependence of the quasi-linear scattering rates on the wave normal distribution of chorus waves, J. Geophys. Res., 114, A11205, doi:10.1029/2009JA014223.

Shprits, Y. Y., R. M. Thorne, R. B. Horne, S. A. Glauert, M. Cartwright, C. T. Russell, D. N. Baker, and S. G. Kanekal (2006a), Acceleration mechanism responsible for the formation of the new radiation belt during the 2003 Halloween solar storm, Geophys. Res. Lett., 33, L05104, doi:10.1029/2005GL024256.

Shprits, Y. Y., W. Li, and R. M. Thorne (2006b), Controlling effect of the pitch angle scattering rates near the edge of the loss cone on electron lifetimes, J. Geophys. Res., 111, A12206, doi:10.1029/2006JA011758.

$\mathrm{Su}, \mathrm{Z}$., H. Zheng, and S. Wang (2009), Evolution of electron pitch angle distribution due to interactions with whistler mode chorus following substorm injections, J. Geophys. Res., 114, A08202, doi:10.1029/ 2009JA014269.

Summers, D., B. Ni, and N. P. Meredith (2007), Timescales for radiation belt electron acceleration and loss due to resonant wave-particle interactions: 2. Evaluation for VLF chorus, ELF hiss, and electromagnetic ion cyclotron waves, J. Geophys. Res., 112, A04207, doi:10.1029/2006JA011993.

Tao, X., A. A. Chan, J. M. Albert, and J. A. Miller (2008), Stochastic modeling of multidimensional diffusion in the radiation belts, J. Geophys. Res., 113, A07212, doi:10.1029/2007JA012985.

Tao, X., J. M. Albert, and A. A. Chan (2009), Numerical modeling of multidimensional diffusion in the radiation belts using layer methods, J. Geophys. Res., 114, A02215, doi:10.1029/2008JA013826.

Thorne, R. M., B. Ni, X. Tao, R. B. Horne, and N. P. Meredith (2010), Scattering by chorus waves as the dominant cause of diffuse auroral precipitation, Nature, 467, 943-946, doi:10.1038/nature09467.

Trakhtengerts, V. Y. (1995), Magnetosphere cyclotron maser: Backward wave oscillator generation regime, J. Geophys. Res., 100(A9), 17,205-17,210.

Tsurutani, B. T., and E. J. Smith (1974), Postmidnight chorus: A substorm phenomenon, J. Geophys. Res., 79(1), 118-127.

Varotsou, A., D. Boscher, S. Bourdarie, R. B. Horne, N. P. Meredith, S. A Glauert, and R. H. Friedel (2008), Three-dimensional test simulations of the outer radiation belt electron dynamics including electron-chorus resonant interactions, J. Geophys. Res., 113, A12212, doi:10.1029/ 2007JA012862.

Wrenn, G. L., J. F. E. Johnson, and J. J. Sojka (1979), Stable 'pancake' distributions of low energy electrons in the plasma trough, Nature, 279 512-514, doi:10.1038/279512a0.

Xiao, F., Z. Su, H. Zheng, and S. Wang (2009), Modeling of outer radiation belt electrons by multidimensional diffusion process, J. Geophys. Res., 114, A03201, doi:10.1029/2008JA013580.

Xiao, F., Z. Su, H. Zheng, and S. Wang (2010), Three-dimensional simulations of outer radiation belt electron dynamics including cross-diffusion terms, J. Geophys. Res., 115, A05216, doi:10.1029/2009JA014541.

R. B. Horne and N. P. Meredith, British Antarctic Survey, Madingley Road, Cambridge CB3 0ET, UK

W. Li, B. Ni, X. Tao, and R. M. Thorne, Department of Atmospheric and Oceanic Sciences, University of California, 7115 Math Sciences Bldg., 405 Hilgard Ave., Los Angeles, CA 90095, USA. (xtao@atmos.ucla.edu) 